\title{
GEOMETRY OF THE LHC SHORT STRAIGHT SECTIONS BEFORE INSTALLATION IN THE TUNNEL: RESULTING APERTURE, AXIS AND BPM POSITIONING
}

\author{
D. Missiaen, P. Bestmann, M. Buzio, S. Fartoukh, M. Giovannozzi, B. Jeanneret, A. Lombardi, Y. \\ Papaphilippou, J. C. Perez, S. Pauletta, H. Prin, E. Wildner, CERN, Geneva, Switzerland.
}

\section{Abstract}

The Large Hadron Collider Short Straight Sections (SSS) are currently being installed in their final position in the accelerator tunnel. For all the SSSs, both those in the regular arcs as well as those in the insertion regions, magnetic axis and geometric measurements are made at different steps of their assembly. These stages range from production in the industry to the cryostating at CERN, as well as during and after cold tests or during installation of the BPM/beam screen assembly. The results of the geometry at the various production stages by means of different procedures and analysis tools are reported and discussed in details in this paper.

\section{THE ARC SHORT STRAIGHT SECTIONS}

The arc quadrupoles, also called Short Straight Sections (SSS), have a magnetic length of $3.1 \mathrm{~m}$ and are of 60 different variants depending on the correctors inside the SSS cryostat, e.g. sextupoles, octupoles, quadrupoles. Each cold mass SSS is constituted by an inertia tube containing a MQ and the above mentioned correctors. All the 360 SSS are built in industry.

Some other 32 quadrupoles for the insertion regions are built by the same manufacturer using the same assembly technique. These magnets are made of a MQ and one or two MQTL. They will be treated together with the Arc SSS in this chapter.

\section{Geometrical Measurement in Industry}

During the assembly phase of the cold mass of these SSS in the industry, the extremities of the various tubes, and in particular the cold bores and the Beam Positioning Monitor (BPM) supports, are aligned using the laser tracker technique with respect to the mechanical axis of the inertia tube.

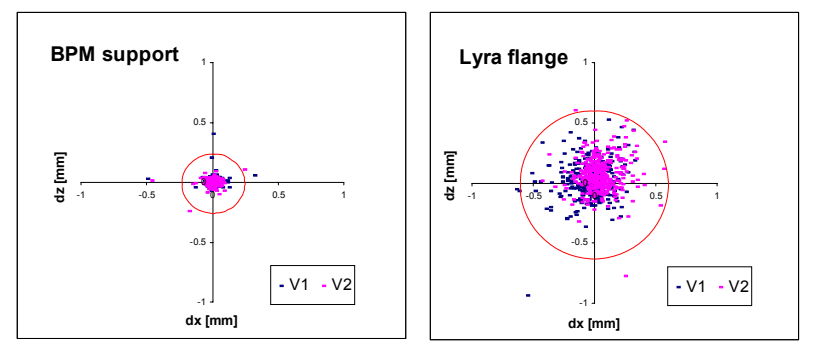

Figure 1: Position of BPM support and lyra flange.

Figure 1 shows that the position of the BPM support and the lyra flange are almost within the specifications of $0.2 \mathrm{~mm}(3 \sigma)$ on the BPM side and $0.6 \mathrm{~mm}(3 \sigma)$ on the lyra side. The standard deviation are $0.03 \mathrm{~mm}$ in $\mathrm{x}$ and $\mathrm{z}$ on the BPM side and $0.14 \mathrm{~mm}$ in $\mathrm{x}$ and $\mathrm{z}$ on the lyra side.

For the insertion quadrupoles MQ/MQTL, the gap between the laminations and the cold bore tube is bigger inside the MQTL, which means that the cold bore is not as representative of the magnet axis as for the Arc SSS. These magnets being also more demanding in term of aperture, it was therefore decided to control the geometry of the cold bore tubes at this stage of the assembly in industry and in particular the position of the flanges were optimised in collaboration with CERN's experts.

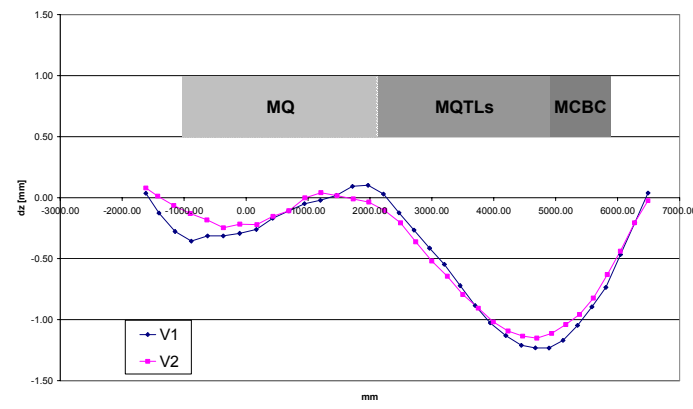

Figure 2: Vertical profile of MQ/MQTL SSS509 in industry.

\section{Geometrical Measurement at CERN Reception}

The insertion quadrupoles MQ/MQTL are usually installed in machine slots where the aperture constraints are very tight. In case of problems with the magnet geometry, the decision on changing the nominal slot should be taken before the hardware type is fully specified. Hence, it was decided to analyze the geometry measurements in industry to verify whether the cold mass fitted the aperture requirements for the nominal slot. In case, corrections were required and their impact further verified. Then, at CERN reception the cold mass was checked for movements during the transport and the slot confirmed.

\section{Fiducialisation Measurement}

The fiducialisation is the operation during which the position of the alignment fiducials, located on the cryostat, are measured with respect to a reference axis. Initially, the reference axis was chosen to be the magnetic axis at cold temperature. But for practical reasons and in order to optimise the mechanical aperture, the reference axis was chosen to be the mechanical axis of the cold bore tubes.

This measurement is done using a laser tracker aiming at a corner cube located inside a "geomole" centring itself 
automatically in the cold bore tubes. The measurements are taken from six positions of the laser tracker, one in front of each tube at each extremity of the SSS and two for determination of the fiducials. All the measurements are linked by network points. The whole accuracy of the measurement is $0.1 \mathrm{~mm}(1 \sigma)$ [1]. This technology coupled with a rigorous method provides with measurement redundancy, a key point for the quality control process.

During this operation, the position of all the tubes at each end of the SSS is measured and corrections are done when the position is not within the tolerance.

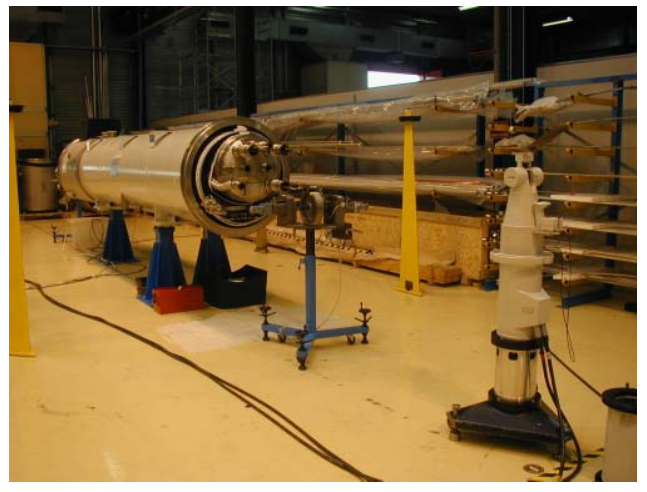

Figure 3 : Fiducialisation stand.

Despite the fact that the reference co-ordinate systems in industry and at this stage are not the same, the deviations of the position of the cold bore tubes is very small between both measurements : the average $\mathrm{dx}$ is $0.01 \mathrm{~mm}(0.17 \mathrm{~mm}$ at $1 \sigma)$ and average $\mathrm{dz}$ is $+0.20 \mathrm{~mm}$ $(0.20 \mathrm{~mm}$ at $1 \sigma)$. This gives a good confidence on the quality and reproducibility of the measurements.

A complementary magnetic axis measurement was done on the first 200 Arc SSS. This measurement is done with the AC mole [2], a rotating mole being measured by the same laser tracker located at the same position as mentioned above. The deviation of the magnetic axis with respect to the reference axis is $0.07 \mathrm{~mm}(1 \sigma)$ in the horizontal direction and $0.12 \mathrm{~mm}(1 \sigma)$ in the vertical one. It was therefore decided to stop this measurement on this type of SSS.

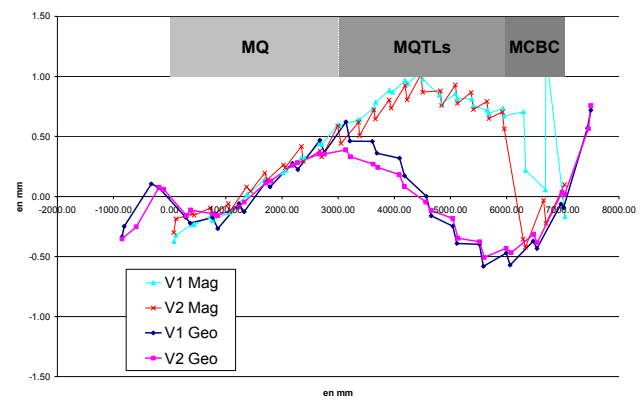

Figure 4: Vertical geometrical and magnetic axis of insertion SSS509.

The magnetic axis measurement was done on all the insertion SSS. Figure 4 shows some important deviations between both axes in particular in the vertical direction. This is due, as explained before, by the larger gap between laminations and cold bore tubes in the MQTL.

\section{Single Stretched Wire Measurement}

The magnetic axis was also measured at warm for 28 SSS and at cold temperature for 11 of them and related to the fiducials using the Single Stretched Wire (SSW) technique [3] in order to cross check with the $\mathrm{AC}$ mole measurements and also to determine the cold-warm shrinkage. The transfer of the magnet axis to the alignment fiducials is also done using a laser tracker.

Table 1: deviations between magnetic axis measurements at warm done with $\mathrm{AC}$ mole and SSW.

\begin{tabular}{|c|c|c|c|c|c|}
\hline \multicolumn{2}{|c|}{ Dx(m) } & \multicolumn{2}{c|}{ Dz (mm) } & \multicolumn{2}{c|}{ Roll angle (mrad) } \\
\hline Mean & Stdev & Mean & Stdev & Mean & Stdev \\
\hline-0.08 & 0.42 & 0.04 & 0.43 & 0.16 & 0.48 \\
\hline
\end{tabular}

No horizontal movement and no rotation were measured during the cool down but an expected average vertical shrinkage of $1.35 \mathrm{~mm}$ was registered. This value is taken into account during the alignment operation in the tunnel.

\section{THE SPECIAL SHORT STRAIGHT SECTIONS}

The superconducting quadrupoles for the insertion regions comprise several superconducting quadrupoles of the MQM or MQY types arranged to give the necessary field strength. In total, 82 Special Short Straight Sections (SSSS) with 31 types of cold masses arranged in 11 families with 6 different lengths from 5.3 up to $11.3 \mathrm{~m}$ [4] are assembled at CERN using quadrupole magnets and dipole correctors manufactured in industry.

\section{Geometrical Measurement During Assembly at CERN}

The individual magnets and correctors are assembled inside a cold mass closed by two half shells welded together under a press, the same technique that was applied for the LHC arc dipoles. The straightness of this assembly, not as rigid as an inertia tube, is carefully monitored by a laser tracker during the production and geometry measurements are done at every step, in particular the positioning of the cold bore tube at the extremities.

The spread of the measurements inside the cold bore tubes is $0.21 \mathrm{~mm}$ in the horizontal plane and $0.34 \mathrm{~mm}$ in the vertical plane, the difference between the two planes coming mostly from the general tendency of the beam tube to sag inside the correctors. The extremities of the beam tubes are aligned to better than $\pm 0.2 \mathrm{~mm}$ on the BPM side and $\pm 0.4 \mathrm{~mm}$ on the lyra side [4].

\section{Fiducialisation Measurement}

The fiducialisation was done for all these SSSS with the same technique, instrumentation and reference axis as for 
the Arc SSS. The cold bore tubes deviations to nominal in both planes are Gaussian and spread is $0.24 \mathrm{~mm}$ in horizontal and $0.33 \mathrm{~mm}$ in vertical.

The magnetic axis was also measured with the $\mathrm{AC}$ mole on all of these magnets.

Table 2: deviations between the reference axis and the magnetic axis measured with $\mathrm{AC}$ mole.

\begin{tabular}{|c|c|c|c|c|c|}
\hline \multicolumn{2}{|c|}{ Dx $(\mathrm{mm})$} & \multicolumn{2}{c|}{ Dz $(\mathrm{mm})$} & \multicolumn{2}{c|}{ Roll angle (mrad) } \\
\hline Mean & Stdev & Mean & Stdev & Mean & Stdev \\
\hline-0.03 & 0.2 & 0.34 & 0.34 & - & - \\
\hline
\end{tabular}

The position of the cold bore tubes at each extremity of the cold mass was measured. Tolerance is $0.5 \mathrm{~mm}$ for the BPM support and $0.87 \mathrm{~mm}$ for the lyra side. The tubes out of tolerance were corrected or the magnet aligned in the tunnel with a shift.

\section{Single Stretched Wire Measurement}

Nine SSSS were measured using the SSW technology at warm and cold temperature. The deviation (Table 3 ) in $\mathrm{z}$ is coherent with the value measured with the $\mathrm{AC}$ mole. The vertical shrinkage is $1.15 \mathrm{~mm}$ or $1.25 \mathrm{~mm}$ depending on the structure of the supporting posts.

Table 3: Deviations between the reference axis and SSW measurements at warm.

\begin{tabular}{|c|c|c|c|c|c|}
\hline \multicolumn{2}{|c|}{ Dx(mm) } & \multicolumn{2}{c|}{ Dz (mm) } & \multicolumn{2}{c|}{ Roll angle (mrad) } \\
\hline Mean & Stdev & Mean & Stdev & Mean & Stdev \\
\hline-0.03 & 0.16 & 0.45 & 0.5 & - & - \\
\hline
\end{tabular}

\section{THE SHIFTS}

In order to optimise the machine aperture and to put back the magnets on tolerance, shifts and rotations were applied for the arc SSS, using a special java based tool [5] enabling visualisation of all relevant geometry measurements including aperture tolerances. Shifts were applied on $30 \%$ of the SSS, mainly in the vertical direction (Fig.5) where the aperture is most critical [6] and rotations on $20 \mathrm{SSS}$. Only twelve SSS were found out of tolerance and flagged as potential aperture restrictions during machine commissioning and operation.

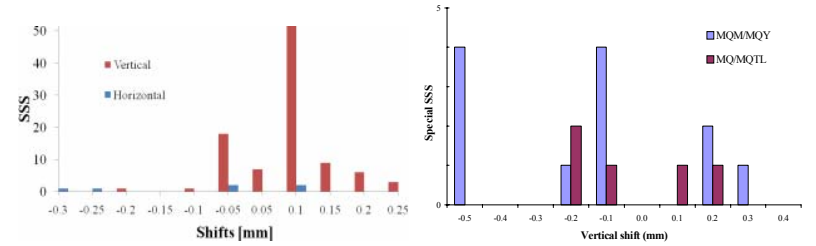

Figure 5: Shifts of arc SSS (left) and vertical installation shift (right) for insertion quadrupoles.

The shifts of the Special SSS were computed with a one-by-one evaluation of the geometrical data. In most of the case the shifts are in the vertical plane (Fig. 5): in one case (out of $32 \mathrm{MQ} / \mathrm{MQTL}$ assemblies) and one (out of $82 \mathrm{MQM} / \mathrm{MQY}$ assemblies) horizontal shifts were applied with maximum amplitude of $0.15 \mathrm{~mm}$. Also, installation roll was computed, partly to improve the aperture, but mainly to compensate for a natural roll of the assembly, which would generate linear coupling in the machine. The values are comprised between -1.3 and $1.5 \mathrm{mrad}$.

\section{DRIFT TUBE AND BPM ALIGNMENT}

The SSS beam screen is terminated at the connection side by a block BPM/drift tube in a rigid assembly. The beam screen once inserted in the cold bore tube, the BPM button has to be installed parallel to the magnet plane and welded to its support while the extremity of the drift tube is positioned within a tolerance of $1.3 \mathrm{~mm}$ for aperture and interconnection reasons. Therefore the positioning of the drift tube was monitored by a laser tracker, relocated in the reference axis system. During the same operation, the position of the BPM button, requested for the machine operation and its roll angle are carefully measured.

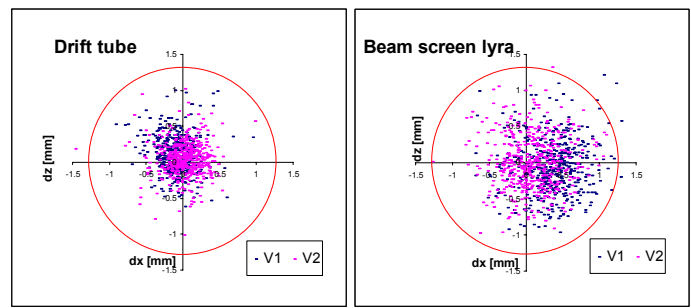

Figure 6: Drift tube and lyra side beam screen position.

\section{CONCLUSIONS}

Many measurements were done during the assembly of the complex LHC quadrupoles using the laser tracker technology and different types of moles. These measurements permitted to produce objects within tolerance for the interconnection and aperture, to know the fiducialisation parameters and shifts indispensable for the alignment in the tunnel as well as the position of the beam monitors requested for machine operation.

\section{REFERENCES}

[1] P. Bestmann, CERN, "Final geo-magnetic Fiducialization of the LHC Short Straight Sections", LHC-G-TP-0006 v1, EDMS 790018.

[2] Marco Buzio et al "A Device to Measure Magnetic and Mechanical Axis of Superconducting Magnets for the Large Hadron Collider at CERN", IEEE Instrumentation and Measurement Technology Conference, Sorrento, Italy, 2006.

[3] J. Di Marco et al., " Field alignment of quadrupole magnets for the LHC interaction regions", IEEE Trans. Appl. Supercond, vol. 10, Jan. 2000, pp. 127130.

[4] H. Prin et al, "Production of the Superconducting Matching Quadrupoles for the LHC insertions", MT19.

[5] J.Beauquis, http://project-lhc-shiftsss.web.cern.ch /project-lhc-shiftsss/

[6] J.B. Jeanneret, LHC Report 1007, June 2007 "Geometrical tolerances for the qualification of LHC magnets" 